\title{
Employing Code Domain for Contention Resolution in Optical Burst Switched Networks With Detailed Performance Analysis
}

\author{
Mohammad Y. S. Sowailem, Student Member, IEEE, Mohamed H. S. Morsy, Student Member, IEEE, and \\ Hossam M. H. Shalaby, Senior Member, IEEE
}

\begin{abstract}
We propose the implementation of spectral-amplitude-coding optical code division multiple access (SAC-OCDMA) as a contention resolution technique in optical burst switched (OBS) networks. The new system architecture is presented in details where an all-optical methodology for cancelling multiple access interference is proposed. Performance evaluation of the proposed system in both MAC and optical layers is introduced where the overall burst error rate of the system is evaluated in three cases: full, partial, and no code conversion capabilities taking into account the receiver dark current, thermal, and shot noises at the egress nodes. Our results reveal that a considerable improvement in the performance of each core node in the system is achieved by using SAC-OCDMA instead of WDM in the optical layer underneath an OBS based MAC layer. We also conclude that a slight increase in the employed number of code converters enhances the overall system performance noticeably. Finally, optimum values for the number of codes, which lead to minimum overall burst error rate, are reached at different traffic conditions.
\end{abstract} tion.

Index Terms-Code division multiplexing, optical communica-

\section{INTRODUCTION}

$\mathbf{T}$ HE tremendous increase of the data rate demand necessitates utilizing the vast bandwidth available on optical fiber links which makes it obligatory to realize the dream of all optical networks (AONs). One of the approaches that target this goal is a paradigm called optical burst switching (OBS) which was first proposed in literature by Qiao and Yoo [1]. In OBS, switching is made on a burst by burst basis where the burst comprises of a group of aggregated packets having the same destination and class.

One-way reservation protocols such as Just-Enough-Time (JET) [1] and Just-In-Time (JIT) [2] are commonly used for signaling in OBS. In these protocols, for each data burst (DB) transmission, one control packet (CP) is sent on a separate control channel prior to the DB with an offset time equals to the processing time of its corresponding $\mathrm{CP}$ in the intermediate

Manuscript received April 02, 2009; revised June 16, 2009 and July 26, 2009. First published August 28, 2009; current version published October 09, 2009.

The authors are with the Electrical Engineering Department, Faculty of Engineering, University of Alexandria, Alexandria, 21544, Egypt (e-mail: eng.m.y. sowailem@gmail.com; holly_pharaoh@msn.com; eng_m_morsy@alex. edu.eg; shalaby@ieee.org).

Color versions of one or more of the figures in this paper are available online at http://ieeexplore.ieee.org.

Digital Object Identifier 10.1109/JLT.2009.2031125 stages in order to reserve resources for the ensuing DB. In our paper, we propose some modifications to the information carried by the $\mathrm{CP}$ in order to be appropriate with the newly added OCDMA scheme.

The OBS network architecture consists of three components; ingress nodes, core nodes and egress nodes [3]. The DB enters the network through the ingress node after aggregating the data packets with the appropriate assembly algorithm [4], [5], and [6]. While at the core node, the $\mathrm{CP}$ is processed reserving appropriate resources for the upcoming DB and configuring the switch fabric to bypass the DB upon its arrival to the destined port. The egress node is the destination node at which the DB is disassembled into original packets, each of which is directed to its own destination.

Although one-way reservation protocols are characterized by small latency, the major hurdle that hinders these protocols is the unacceptable burst loss probability due to probable contentions. To reduce this dropping probability, various contention resolution techniques were proposed which depend on multiplexing the contending DBs in either wavelength, time or space domain using either wavelength converters, fiber delay lines (FDLs) or deflection routing successively.

In [7], Kamakura et al. proposed using OCDMA as a contention resolution scheme where the multiplexing is done in the code domain. Specifically, Kamakura et al. presented a new QoS differentiation scheme by embedding codewords with priority-dependent weights in a single used optical orthogonal code (OOC). However, the paper did not give a detailed description for the architecture of the proposed system. Furthermore, the DB in their system experiences multiple access interference (MAI) from other users on the same OOC which negatively affects the throughput.

The aim of this paper is to propose the implementation of spectral-amplitude-coding optical code division multiple access (SAC-OCDMA) as a new contention resolution technique in OBS. The main idea in the newly proposed system (OBS/SAC-OCDMA) is to increase the number of available resources (codes) for reservation instead of the more limited resources (wavelengths) while using OBS/WDM. The SAC-OCDMA scheme is characterized by the zero MAI feature when using the balanced receiver structure [8]. Also, the SAC-OCDMA system remains feasible for implementation using the FBG-based encoder/decoder [9]. Inspired by the zero MAI feature and the possibility to increase the code length, hence accommodating more simultaneous users, we propose the 
new system (OBS/SAC-OCDMA) in order to reduce the overall burst error rate of the OBS network by reducing the probability for contention to occur over the case when WDM is used in the optical layer (OBS/WDM) where there is an upper bound to the number of wavelengths available in a given spectral region. Next, we thoroughly study the performance of the proposed OBS/SAC-OCDMA system in both MAC and optical layers. In addition, we evaluate the effect of adding code converters in the resources of the core node on the overall performance of the system. Finally, throughout the performance evaluation of the proposed system, we target the optimum number of codes with which the system should be designed at different traffic conditions in order to achieve the minimum possible value for the overall burst error rate.

The remainder of this paper is organized as follows. In Section II, we present a detailed description for our proposed system (OBS/SAC-OCDMA). Section III is devoted for the performance evaluation of the proposed system. In Section IV, numerical results for the derived performance measures are introduced. Finally, we give our conclusion in Section V.

\section{SYSTEM DESCRIPTION}

\section{A. Basics of OBS With SAC-OCDMA}

In the OBS/WDM network, the CP contains two main fields of information; the wavelength on which the upcoming DB will arrive, and the destination to which this DB should be forwarded. These two fields of information are essential for the core node to bypass the DB to the destined output port.

However, in our proposed OBS/SAC-OCDMA system, we rely on multiplexing different $\mathrm{DBs}$ in the code domain rather than on the wavelength domain in the ordinary OBS/WDM system. Thus, the CP should not contain information about the wavelength on which the DB is transmitted; rather it should contain information about the code used to mask the spectrum of the source during the transmission of its corresponding DB. Based on the code, and destination information in the $\mathrm{CP}$, the core node will be capable of forwarding the DB to the proper output port in the so called code-based forwarding process which is explained in details later in this section.

\section{B. Ingress Node Description}

In our newly proposed system, the ingress node has an additional mission rather than computing the offset time and assembling the packets into DBs; it should (through the burst scheduler in Fig. 1) assign a code to the DB before transmission and adjust the spectral encoder to mask the spectrum produced by the broadband amplified spontaneous emission (ASE) source with the assigned code. This broad bandwidth $\Delta \nu$ is divided into $l$ slices to be encoded according to the code assigned by the scheduler. We assume that the power distribution of the ASE source is flat over the spectrum which means that $P_{\text {slice }}=P_{\text {ASE_ingress }} / l$, where $P_{\text {ASE_ingress }}$ is the total power emitted from the ASE source placed at the ingress node and $P_{\text {slice }}$ is the power of one spectral slice. The codes deployed in our system are generated from a Hadamard matrix of size $l \times l$. We denote by $N$ the number of codes that are currently in use in the system which has a maximum value of $l-1$ where all

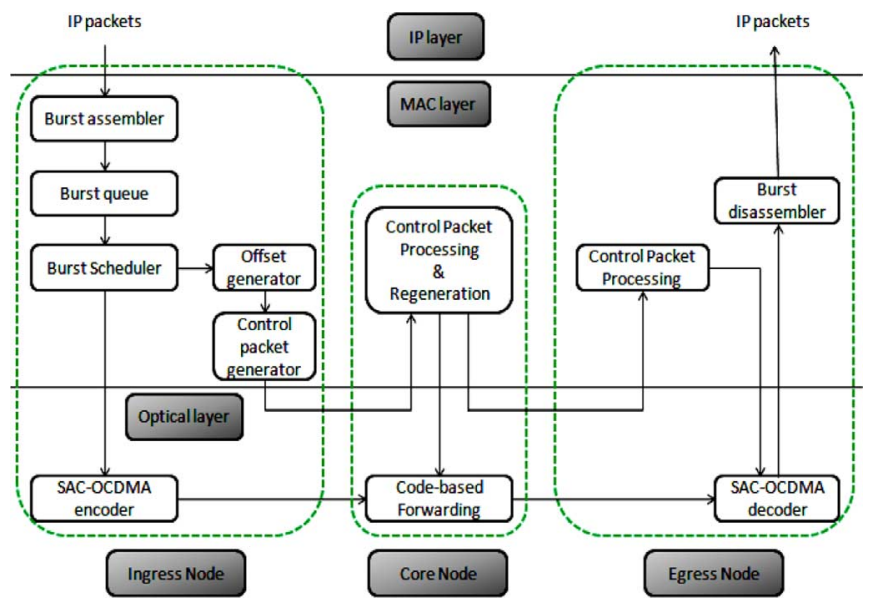

Fig. 1. Layers of the OBS/SAC-OCDMA system.

the rows of the Hadamard matrix are assigned to different DBs except the first row.

In Fig. 2(a), a schematic diagram shows the spectral encoder at the ingress node where the DB is encoded with one of the $N$ codes using the tunable chirped fiber Bragg grating (FBG1) [9]. In the encoding process of the DB, FBG1 is tuned to mask the spectrum produced by the ASE source according to the assigned code. This is done via passing certain slices in the spectrum according to the mark positions of the assigned code while reflecting the slices that correspond to the space positions in the code, i.e., the spectral components that bypass FBG1 are the ones that are transmitted. The switch shown in Fig. 2(a) performs the On-Off Keying (OOK) of the input data by bypassing the spectrum of the ASE source to FBG1 when a data ' 1 ' is sent and prevents the spectrum from passing to FBG1 when a data ' 0 ' is sent. Thus, the encoder emits a signal power $l P_{\text {slice }} / 2$ when a data ' 1 ' is sent while it produces no power when a data ' 0 ' is sent. It should be noted that we deploy OOK instead of complementary keying in our system although the later has a 3 $\mathrm{dB}$ advantage over OOK. This is because using complementary keying imposes implementing an additional FBG at each encoder in the whole system increasing its overall cost; however, complementary keying can still be easily added to our system.

\section{Core (Intermediate) Node Description}

According to Fig. 1, the core node operation lies in both the MAC and optical layers. Operation in the MAC layer involves $\mathrm{CP}$ processing and extracting the information required for bypassing the corresponding DB (code assigned, time of arrival, and destination). However, operation in the optical layer involves the reception and retransmission of the received spectrally encoded data, MAI cancelation process and power adjustment to perform the code-based forwarding process.

It is assumed that the core node has equal number of $M$ input and output ports as shown in Fig. 3(a). A splitter is placed at each input port to split the received signal over $N$ paths. Each path contains an all-optical MAI canceller which aims to extract the desired DB, which has been assigned a specific code that is dedicated to this path, from its interferers. The all-optical MAI canceller comprises of two chirped FBGs (FBG2 and 


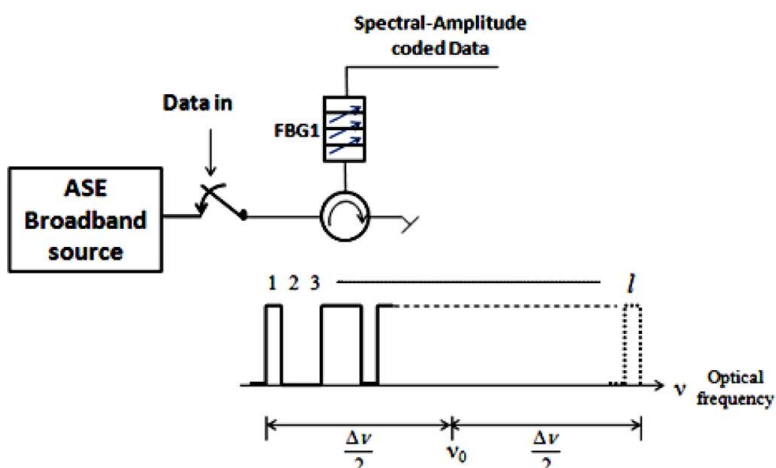

(a)

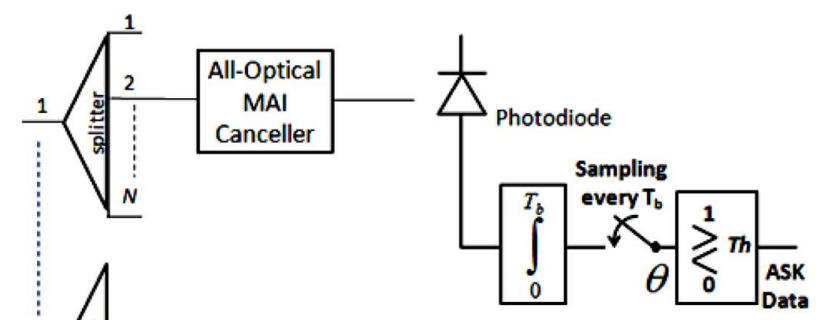

(b)

Fig. 2. Architecture of an edge (ingress/egress) node in OBS/SAC-OCDMA system.

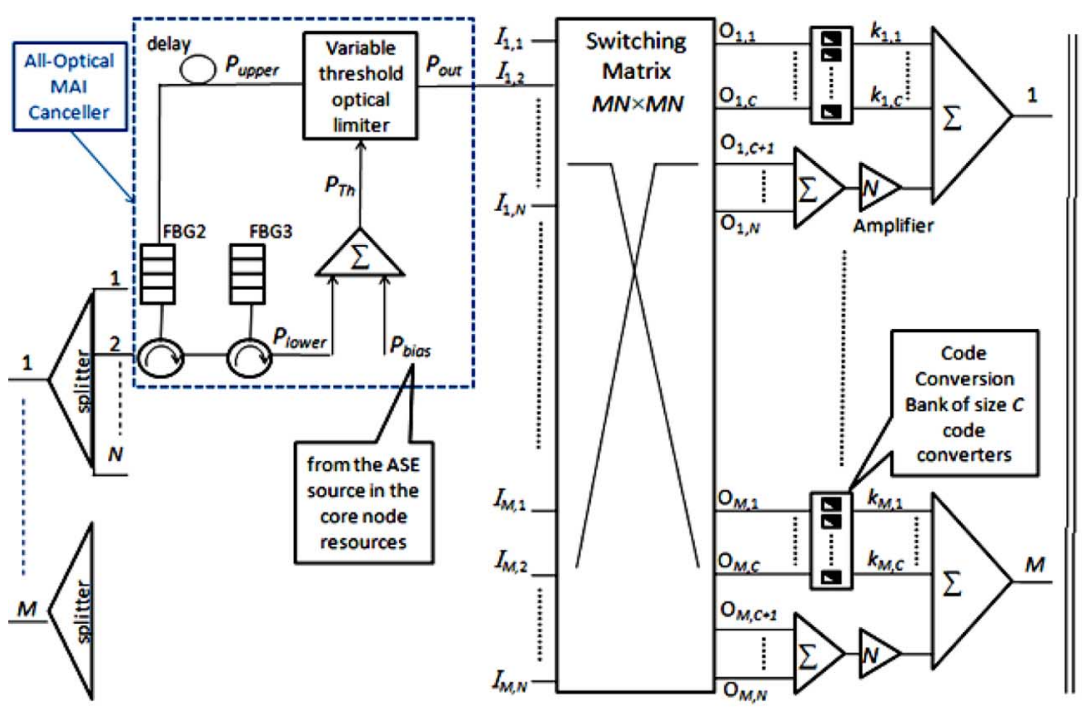

(a)

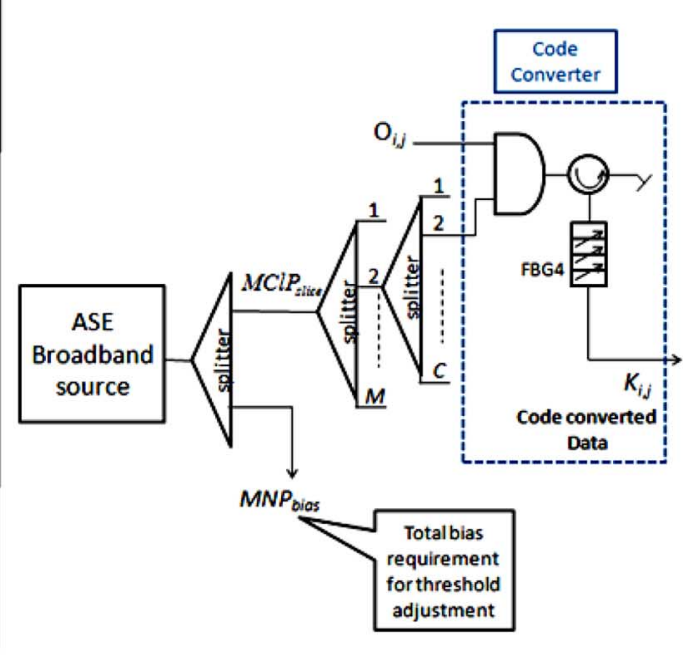

(b)

Fig. 3. Architecture of a core node in OBS/SAC-OCDMA system.

FBG3) where FBG2 is designed to pass the part of the spectrum that corresponds to the marked positions of the specific code and reflect the power on the space positions (the complement) of the DB's code, while FBG3 is designed to align the spectrum slices reflected from FBG2 in time. The delay in the upper branch is introduced to compensate the round-trip delay introduced by FBG2 and FBG3, hence realign the two spectra on the two branches in time.

The key idea of the reception of a spectrally encoded signal in an SAC-OCDMA system is to make the interferers cancel themselves, which is based on the fact that the Hadamard codes have the property that an interfering code overlaps with the desired code and its complement in exactly $l / 4$ mark positions, where $l$ is the code length. Applying this property, we can formulate the power levels in the upper and lower branches, shown in Fig. 3(a), $P_{\text {upper }}$ and $P_{\text {lower }}$ respectively as follows:

$$
\begin{aligned}
P_{\text {upper }} & = \begin{cases}\frac{l P_{\text {slice }}}{4 N}\left(N_{\text {interf }}+2\right) & \text { '1'sent } \\
\frac{l P_{\text {slice }}}{4 N} N_{\text {interf }} & \text { ' }\end{cases} \\
P_{\text {lower }} & =\frac{l P_{\text {slice }}}{4 N} N_{\text {interf }}
\end{aligned}
$$

where $N_{\text {interf }}$ denotes the number of interferers. By making use of that property, the MAI will be eliminated by subtracting the two signal powers on the two branches $P_{\text {upper }}$ and $P_{\text {lower }}$. The key component in the all-optical MAI canceller is the variable threshold optical limiter whose functionality is equivalent to this subtraction process where it hard limits $P_{\text {upper }}$ according to the variable threshold power $P_{\text {Th }}$ which is controlled by $P_{\text {lower }}$. This implies that the optimum value of $P_{\text {Th }}$ should be set at the middle of the range between $P_{\text {upper }}$ and $P_{\text {lower }}$, i.e., $P_{\text {Th }}=$ $\left(l P_{\text {slice }}\right) /(4 N)\left(N_{\text {interf }}+1\right)$. Hence, an active power $P_{\text {bias }}$ equal to $l P_{\text {slice }} / 4 N$ introduced by an ASE source placed at the core node shown in Fig. 3(b) is combined with $P_{\text {lower }}$ to adjust its value to reach the optimum $P_{\mathrm{Th}}$. Specifically, the required characteristics of the variable threshold optical limiter is given as follows:

$$
P_{\text {out }}=\left\{\begin{array}{ll}
\frac{l P_{\text {slice }}}{2 N} & P_{\text {upper }}>P_{\mathrm{Th}} \\
0 & P_{\text {upper }}<P_{\mathrm{Th}}
\end{array} .\right.
$$

After the MAI cancelation process, the output from the all-optical MAI canceller is introduced to the non-blocking $M N \times$ $M N$ switching matrix. In order to resolve the probable contention at each output port, we deploy a code conversion bank 
that comprises of $c$ code converters, where $c<N$, ready to be shared by the DBs forwarded to this output port, i.e., a SharePer-Line (SPL) strategy is adopted as shown in Fig. 3(a). The operation of each code converter in the bank is separately illustrated in Fig. 3(b) where it acts as a spectral encoder that retransmits the data on the output line $\mathrm{O}_{i, j}$ of the switching matrix, where $i \in\{1,2, \ldots, M\}$ and $j \leq \mathrm{c}$, by encoding the spectrum of the ASE source through the AND gate [10] controlled by the data on the output line $\mathrm{O}_{i, j}$ with a free code to which the original DB's code shall be converted. Finally, we should note that the power level at the output of the code converter equals $l P_{\text {slice }} / 2$ given that the data on $\mathrm{O}_{i, j}$ is ' 1 ' while no power is emitted if the data on $\mathrm{O}_{i, j}$ is ' 0 '.

In the above discussion, the number of code converters is limited to $c$, where $c<N$, which means that partial code conversion capability is employed. This is justified by two restrictions that sophisticate the implementation of large number of code converters in the bank. First, the power budget needed from the ASE source in the core node is proportional to $c$. Second, the cost of implementation of such an advanced technology of the tunable chirped FBGs also increases with increasing the value of $c$. Another issue regarding the partial code conversion is that the power level at each of the output lines of the code converters $K_{i, j}$ is different from that on the output lines of the switching matrix $\mathrm{O}_{i, j}$ for $c<j \leq N$. Thus, an amplifier is introduced to the lines $\mathrm{O}_{i, j}$, where $c<j \leq N$, that did not undergo the code conversion process in order to boost them to the power level at $K_{i, j}$. Finally, the total power requirement from the ASE source that should be placed at the core node equals $c M l P_{\text {slice }}+M N P_{\text {bias }}$.

Clearly, the purpose behind the code-based forwarding process performed in the core node is to be able to separate a DB on a distinct code from its interferers, i.e., the DB is properly extracted in order to be able to forward this DB based on its code to its destined output port with the appropriate power level. The interference cancellation performed via the all-optical MAI canceller reduces the possibility for blocking to occur as it leaves more free codes for reservation on each of the $M$ output ports.

\section{Egress Node Description}

It is assumed that the egress node has $M$ input ports; each port is introduced to a splitter which splits the input power to $N$ paths, each of which is dedicated to a specific code reception. The cancellation of the MAI is performed optically through the proposed all-optical MAI canceller and the receiver makes the decision only in the electronic domain. After the MAI canceller, the output power that strikes the photodetector in the egress node is either $l P_{\text {slice }} / 2 N$ if a data ' 1 ' is sent or zero if a data ' 0 ' is sent. Finally, a decision can be made using the circuitry shown in Fig. 2(b) via comparing the sampled output from the integrate and dump circuit against a certain threshold value. After every bit in the DB is received, the DB is disassembled back to its original data packets, each of them can be directed to its own destination. However, the decision made in the electronic domain still might be erroneous due to the existence of the photodetector thermal, shot and dark current noises as will be handled later in the performance evaluation of the system. Finally, we should note that all FBGs used in our proposed system architecture are assumed ideal in the sense that they do not possess any physical imperfections that would affect the number of simultaneous users supported.

\section{Performance Evaluation of the OBS/SAC-OCDMA SYSTEM}

As shown in Fig. 1, the operation of the OBS/SAC-OCDMA system involves both the MAC and optical layers. Hence, for a DB to be correctly received, success should be achieved by the DB while transmission in the optical layer provided that its corresponding $\mathrm{CP}$ has already succeeded in the MAC layer.

\section{A. Performance of the OBS/SAC-OCDMA System in the MAC Layer}

As mentioned earlier, for a DB to be received in success at its destination (egress) node, its corresponding $\mathrm{CP}$ should successfully reserve the appropriate resources at all intermediate (core) nodes that lie on its route extending from the source (ingress) node to the destination (egress) node. To evaluate the performance of the OBS/SAC-OCDMA system in the MAC layer, we study the performance of a single core node in the CP path by calculating the per-node burst loss probability $P_{\text {Node-Loss }}$ from which we calculate the MAC burst loss probability $P_{\mathrm{MAC}-\mathrm{Loss}}$ which measures the overall performance of all nodes that lie on the path between the ingress and egress nodes.

1) Model Assumptions: We perform a Markovian analysis to characterize the performance of a single core node. This analysis is carried out under the following assumptions.

- We assume that the destination for an incoming burst to the core node is uniformly distributed among all available output ports $(1: M)$. Thus, it is sufficient to model the behavior of a single output port.

- A core node is assumed to have the following resources:

i) A single wavelength around which the ASE broadband source emits its light. This single wavelength corresponds to the center frequency $\left(\nu_{0}\right)$ of the coded spectrum as shown in Fig. 2(a).

ii) No fiber delay lines for contention resolution.

iii) A number of $N$ codes that can be used to serve the incoming burst arrivals.

iv) A code converter bank at every output port, each of which comprises of $c$ code converters shared between DBs forwarded to this specific output port, where $c \in$ $\{1,2, \ldots, N\}$, i.e., a Share-Per-Line (SPL) strategy is adopted. Each code converter can convert an arriving $\mathrm{DB}$ on a given code to any other free code. We define the code conversion capability as $\gamma \stackrel{\text { def }}{=}$ $(c) /(N)$ in order to discriminate between three different cases. The two limiting cases when $\gamma=0$ and $\gamma=1$ represent the cases of no and full code conversion respectively, whereas, if $0<\gamma<1$, the node has partial code conversion capability.

- Incoming DBs are assumed to arrive at the node according to a Poisson process with a mean arrival rate $\lambda$ bursts/seconds. In addition, we assume the code on which an incoming DB arrives is uniformly distributed over $(1: N)$. 
Moreover, the service time of an incoming burst is assumed to have an exponential distribution with a mean $1 / \mu$ seconds which is equal to the average duration of the DB.

2) Model Equations for the Limiting Cases: No and Full Code Conversion Capability $(\gamma=0 \& \gamma=1)$ : For the case of full code conversion $(\gamma=1)$, each output port can be safely modeled as an $\mathrm{M} / \mathrm{M} / N / N$ loss system with $N$ servers modeling the $N$ available number of codes. We can obtain the pernode burst loss probability $P_{\text {Node-Loss }}$, which is defined as the probability that a $\mathrm{CP}$ fails to reserve appropriate resources for its corresponding DB at a core node that lies on the path between the ingress and egress nodes, from the well known Erlang-B formula as follows:

$$
P_{\text {Node-Loss }}=\frac{\rho^{N} / N !}{\sum_{i=0}^{N} \rho^{i} / i !}
$$

where the offered load on all the available codes is

$$
\rho \stackrel{\text { def }}{=} \lambda / \mu \text {. }
$$

For the case of absence of code conversion $(\gamma=0)$, each output port can be reasonably modeled as $N$ independent single server $\mathrm{M} / \mathrm{M} / 1 / 1$ loss systems each with an offered load $\lambda / N \mu$. Hence, $P_{\text {Node-Loss }}$ can also be calculated via the Erlang-B formula as follows:

$$
P_{\text {Node-Loss }}=\frac{\rho / N}{1+\rho / N}
$$

3) Model Equations for the Partial Code Conversion Capability $(0 \leq \gamma \leq 1)$ : We are going to evaluate $P_{\text {Node-Loss }}$ in case of partial code conversion $(0<\gamma<1)$ via a Markovian analysis carried out for a certain output port similar to that used in [11]. Let $i(t)$ and $j(t)$ denote the number of codes and the number of code converters inside the output port's converter bank that are in use at time $t$, respectively. The process $X(t)=\{(i(t), j(t)): t \geq 0\}$ is a two dimensional continuous time Markov process (CTMC) on the state space $S=\{(i, j)$ : $0 \leq i \leq N, 0 \leq j \leq \min (i, c)\}$. Now, let us assume that the process $X(t)$ is currently at state at $(i, j)$ time t, and knowing that DB arrivals occur with an average rate $\lambda$ burst/seconds, one of four possible scenarios may take place if a DB arrives:

i) The code on which the DB arrives is currently free on the output port which is an event with a probability $(N-i) / N$ and the DB will be successfully served and the process will jump to state $(i+1, j)$ with a transition rate $\lambda(N-$ i) $/ N$.

ii) The DB's code is currently in use by an another transmission on the desired output with a probability $i / N$, which will lead to one of two possibilities:

- If $j=c$, the DB will be dropped because all code converters in the converter bank are busy which results in no state change;

- If $j<c$, the DB will be successfully served by randomly assigning it to one of the free codes and converting it via one of the free code converters which leads to a state transition to state $(i+1, j+1)$ with a rate $\lambda \cdot(i / N) \cdot(1-\lfloor j / c\rfloor)$. iii) If $i=N$, i.e., all the codes available are already used, then the DB will be blocked leading to no state transitions.

Conversely, if the process $X(t)$ is currently at state $(i, j)$ at time $\mathrm{t}$, given that DB departures occur with a rate $i \mu \mathrm{burst} / \mathrm{sec}-$ onds, one of two possible scenarios may arise if a DB departs the node:

i) The departing DB was using a code converter which is an event with a probability $j / i$, which leads to a transition to state $(i-1, j-1)$ with a rate $i \mu \cdot(j / i)$.

ii) The departing DB was not using a code converter with a probability $(i-j) / i$ leading to a transition to state $(i-$ $1, j)$ with a rate $i \mu(i-j) / i$.

Next, we are going to calculate the generator matrix $Q$ that gives all possible transition rates for this CTMC. For the purpose of generating $Q$, we are going to decompose the state space $S$ into subsets according to the number of codes that are in use. Each subset is called a level and contains all states having the same number of codes currently in use by DBs. The state decomposition into levels is illustrated as follows:

$$
S=\left\{\begin{array}{ccccc}
(0,0) & & & & \\
(1,0), & (1,1) & & & \\
(2,0), & (2,1), & (2,2) & & \\
\vdots & \vdots & \vdots & \ddots & \\
(c, 0), & (c, 1), & (c, 2), & \cdots & (c, c) \\
\vdots & \vdots & \vdots & & \vdots \\
(N, 0), & (N, 1), & (N, 2), & \cdots & (N, c)
\end{array}\right\} \begin{gathered}
\text { level } 0 \\
\text { level 1 } \\
\text { level } 2 \\
\vdots \\
\text { level } c \\
\vdots \\
\text { level } N
\end{gathered} .
$$

It is easy to show that the total number of states, and hence the size of the generator matrix $Q$, is $(N-(c / 2)+1)(c+1)$. Then, it appears that generating $Q$ in a straightforward way is quite cumbersome. Hence, we are going to express $Q$ by dividing it into submatrices $A_{i}, U_{i}$ and $D_{i}$ in a similar way to [11]. The submatrices $A_{i}$ represent the rates of transitions within the same level, and $U_{i}$ represent the rates of transition from a certain level to the higher neighboring level, while $D_{i}$ give the transitions to the lower neighboring level. Hence, the matrix $Q$ can be written as follows:

$$
Q=\left[\begin{array}{cccccc}
A_{0} & U_{1} & & & & \\
D_{0} & A_{1} & U_{2} & & & \\
& D_{1} & A_{2} & \ddots & & \\
& & \ddots & & \ddots & \\
& & & \ddots & \ddots & U_{N} \\
& & & & D_{N-1} & A_{N}
\end{array}\right]
$$

Next $A_{i}, U_{i}$ and $D_{i}$, are expressed in terms of another three matrices $Z_{i}, N_{i}$ and $M_{i}$ as follows:

$$
A_{i}= \begin{cases}-(\lambda+i \mu) I_{i+1} & i<c \\ -(\lambda+i \mu) I_{c+1}+\lambda Z_{i} & c \leq i<N \\ -i \mu I_{c+1} & i=N\end{cases}
$$

where $I_{i}$ is the identity matrix of size $i$, see the equation at the bottom of the page, where $Z_{i}, N_{i}$ and $M_{i}$ are given as follows:

$$
Z_{i}=\left[\begin{array}{cccc}
0 & 0 & \cdots & 0 \\
0 & 0 & \cdots & 0 \\
\vdots & \vdots & \ddots & \vdots \\
0 & 0 & \cdots & i / N
\end{array}\right]
$$




$$
\begin{aligned}
N_{i} & =\left[\begin{array}{cccc}
\frac{N-i}{N} & \frac{i}{N} & & \\
& \frac{N-i}{N} & \frac{i}{N} & \\
& & \ddots & \ddots
\end{array}\right] \\
M_{i} & =\left[\begin{array}{cccc}
i & & & \\
1 & i-1 & & \\
& 2 & i-2 & \\
& & \ddots & \ddots
\end{array}\right] .
\end{aligned}
$$

Next, steady-state probabilities of this CTMC $\pi_{i, j}$ can be found by solving this system of equations:

$$
\bar{\pi} Q=0, \quad \bar{\pi} e=1
$$

where $\bar{\pi}=\left[\pi_{0,0}, \pi_{1,0}, \pi_{1,1}, \pi_{2,0}, \ldots, \pi_{N, c}\right]$ and $e$ is a column vector of ones of size $(N-(c / 2)+1)(c+1)$.

Then, one can easily find the per-node burst loss probability $P_{\text {Node-Loss }}$ from the obtained steady state probabilities as follows:

$$
P_{\text {Node-Loss }}=\sum_{i=0}^{c} \pi_{N, i}+\sum_{j=c}^{N-1} \frac{j}{N} \pi_{j, c} .
$$

Next, we assume the overall network traffic is uniformly distributed across the entire network, hence the offered load $\rho$ and the per-node burst loss probability $P_{\text {Node-Loss }}$ can be safely assumed the same for all nodes. Consequently, one can obtain the MAC burst loss probability $P_{\mathrm{MAC}-\mathrm{Loss}}$, which is the probability that a CP fails in the reservation process at any of the nodes existing in its corresponding DB path, as follows:

$$
P_{\mathrm{MAC}-\text { Loss }}=1-\left(1-P_{\text {Node-Loss }}\right)^{H}
$$

where $H$ is the maximum possible number of core nodes that a path can contain which is the worst case representing the longest possible path that can be established in the network, that results in the highest possible $P_{\text {MAC-Loss }}$. In (5), $P_{\text {Node-Loss }}$ is either given by (2) in case of presence of full code conversion, (3) in case of absence of code conversion or (4) in case of partial code conversion.

\section{B. Performance of the OBS/SAC-OCDMA System in the Optical Layer}

The performance in the optical layer depends mainly on the decision error that might originate from the receiver thermal, shot and dark current noises while photodetecting each bit in the desired DB.

The approach adopted to evaluate the performance of the OBS/SAC-OCDMA system in the optical layer is based on the assumption that success in the reception of a DB is contingent on the success in receiving all its contained bits. Hence, we evaluate the bit error rate, denoted $\mathrm{BER}_{\mathrm{PHY}}$, which is defined as the error rate encountered in the decision while receiving each bit. In order to calculate $\mathrm{BER}_{\mathrm{PHY}}$, we should obtain the probability distribution function $P_{\theta}(\theta)$ of the decision variable $\theta$ shown in Fig. 2(b), which is the accumulated charge after the integrator in Coulombs. After that, one can easily write

$$
\mathrm{BER}_{\mathrm{PHY}}=\frac{1}{2} P_{e / 1}+\frac{1}{2} P_{e / 0}
$$

which can be written as follows:

$$
\mathrm{BER}_{\mathrm{PHY}}=\frac{1}{2} \int_{-\infty}^{\mathrm{Th}} P_{\theta / 1}(\theta) d \theta+\frac{1}{2} \int_{\mathrm{Th}}^{\infty} P_{\theta / 0}(\theta) d \theta
$$

where Th is the decision threshold.

The following are the list of variables with their definitions which are going to be used in the next part of the analysis:

$T_{b}$ :

$R_{b}: \quad$ Bit rate;

$\eta: \quad$ Quantum Efficiency of the photodetectors;

$i_{d}: \quad$ Photodetector dark current;

$h$ : Plank's constant;

$v_{0}: \quad$ Operating frequency;

$e_{0}: \quad$ Electron charge;

$L_{\text {burst }}$ : $\quad$ Average burst length in bits;

$P_{\text {ASE_ingress: }}$ Emitted power from ASE source at the ingress node which also equals $l P_{\text {slice }}$.

We also assume a thermal noise with intensity $N_{0}(\mathrm{~W} / \mathrm{Hz})$, hence the variance of the output thermal noise of an integrator with integration duration of $T_{b}$ seconds will be $\sigma^{2}=N_{0} T_{b} / 2$ [12]. Moreover, we denote by $\lambda_{d}$ the dark current photoelectron rate of the photodetector. Therefore, the mean photoelectron count over a period of $T_{b}$ seconds due to dark current will be $\lambda_{d} T_{b}$. We also denote by $\lambda_{s}$ the photoelectron rate due to incident signal photons. Therefore, the mean signal photoelectron count over a period of $T_{b}$ seconds will be $\lambda_{s} T_{b}$.

Noting that the effects of thermal, shot and dark current noises are taken into consideration, one can easily write

$$
\begin{aligned}
P_{\theta / b}(\theta) & =\sum_{n=0}^{\infty} P(n / b) \frac{e^{\frac{-\left(\theta-n e_{0}\right)^{2}}{2 \sigma^{2}}}}{\sqrt{2 \pi \sigma^{2}}} \\
\text { and } \quad P(n / b) & =\operatorname{Pos}\left(n, b \lambda_{s} T_{b}+\lambda_{d} T_{b}\right)
\end{aligned}
$$

where $b$ is the transmitted bit, $\lambda_{s}=$ $\left(\eta P_{\mathrm{ASE} \text { _ingress }}\right) /\left(2 h \nu_{0} N\right), \lambda_{d}=\left(i_{d}\right) /\left(e_{0}\right), \quad$ and $\operatorname{Pos}(n, X)$ denotes Poisson distribution with a mean $X$.

$$
\begin{aligned}
& U_{i+1}=\lambda \times \begin{cases}\text { upper left }(c+1) \times(c+1) \text { block of } N_{i} & c \leq i, \\
\text { upper left }(i+1) \times(i+2) \text { block of } N_{i} & 0 \leq i<c,\end{cases} \\
& D_{i-1}=\mu \times \begin{cases}\text { upper left }(c+1) \times(c+1) \text { block of } M_{i} & c<i, \\
\text { upper left }(i+1) \times i \text { block of } M_{i} & 1 \leq i \leq c\end{cases}
\end{aligned}
$$


Here, we should note that while calculating $\lambda_{s}$, we divide by 2 , which is justified by the fact that only half of the spectrum is transmitted given ' 1 ' sent while the other half is masked. Another issue while calculating $\lambda_{s}$ is dividing by $N$ in order to consider the effect of the splitting performed at the front end of the egress node. On the other hand, other splitting operations performed at the front end of each core node in the path have no effect on the received signal power because the signal is effectively regenerated at each core node.

Next, we calculate BER $\mathrm{PHY}_{\mathrm{P}}$ by substituting from (7) into (6) and performing the integration as follows:

$$
\begin{aligned}
\operatorname{BER}_{\mathrm{PHY}}= & \frac{1}{2} \int_{-\infty}^{\mathrm{Th}} \sum_{n=0}^{\infty}\left(\frac{G_{1}^{n}}{n !} e^{-G_{1}} \times \frac{e^{\frac{-\left(\theta-n e_{0}\right)^{2}}{2 \sigma^{2}}}}{\sqrt{2 \pi \sigma^{2}}}\right) d \theta \\
& +\frac{1}{2} \int_{\text {Th }}^{\infty} \sum_{n=0}^{\infty}\left(\frac{G_{0}^{n}}{n !} e^{-G_{0}} \times \frac{e^{\frac{-\left(\theta-n e_{0}\right)^{2}}{2 \sigma^{2}}}}{\sqrt{2 \pi \sigma^{2}}}\right) d \theta
\end{aligned}
$$

where $G_{b}=b \lambda_{s} T_{b}+\lambda_{d} T_{b}$

$$
\begin{aligned}
& \mathrm{BER}_{\mathrm{PHY}} \\
& =\frac{1}{2} \sum_{n=0}^{\infty}\left(\frac{G_{1}^{n}}{n !} e^{-G_{1}} \times \frac{1}{2} \operatorname{erfc}\left(\frac{e_{0} n-\mathrm{Th}}{\sqrt{2} \sigma}\right)\right) \\
& \quad+\frac{1}{2} \sum_{n=0}^{\infty}\left(\frac{G_{0}^{n}}{n !} e^{-G_{0}} \times \frac{1}{2} \operatorname{erfc}\left(\frac{\mathrm{Th}-e_{0} n}{\sqrt{2} \sigma}\right)\right) .
\end{aligned}
$$

Finally, we calculate the burst error rate, denoted BurstER $_{\mathrm{PHY}}$, which is defined as the error rate encountered while receiving $\mathrm{DBs}$ due to single bit errors, from $\mathrm{BER}_{\mathrm{PHY}}$ by averaging over all possible values of the burst duration as follows:

$$
\begin{aligned}
\operatorname{BurstER}_{\mathrm{PHY}} & =\int_{0}^{\infty}\left[1-\left(1-\mathrm{BER}_{\mathrm{PHY}}\right)^{R_{b} t}\right] \times \mu e^{-\mu t} d t \\
& =1-\frac{\mu}{\mu-R_{b} \ln \left(1-\mathrm{BER}_{\mathrm{PHY}}\right)} \\
& =1-\frac{1}{1-L_{\mathrm{burst}} \ln \left(1-\mathrm{BER}_{\mathrm{PHY}}\right)}
\end{aligned}
$$

\section{Overall Performance of the OBS/SAC-OCDMA System in Both Layers}

In this part, we study the overall performance of the proposed OBS/SAC-OCDMA system in both MAC and optical layers. This is based on the fact that the success of a DB mandates the success of the DB in the optical layer given that its $\mathrm{CP}$ has succeeded in the MAC layer. After the calculation of the $M A C$ burst loss probability $P_{\mathrm{MAC}-\mathrm{Loss}}$ from (5) and the burst error rate BurstER $\mathrm{PHY}_{\mathrm{P}}$ from (9), one can get the overall burst error

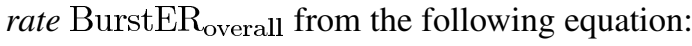

BurstER $_{\text {overall }}=1-\left(1-P_{\mathrm{MAC}-\text { Loss }}\right)\left(1-\right.$ BurstER $_{\mathrm{PHY}}$.

\section{Comparison Between OBS/SAC-OCDMA and OBS/WDM Systems}

By employing the results obtained in (3) and (4), a comparison between the newly proposed OBS/SAC-OCDMA system and the ordinary OBS/WDM system in terms of the MAC pernode burst loss probability $P_{\text {Node-Loss }}$ can be held. We should note that (3) and (4) can be used to obtain $P_{\text {Node-Loss }}$ for the OBS/WDM system similar to the OBS/SAC-OCDMA system by just replacing the number of codes $N$ with the number of wavelengths $N_{w}$. In order to make the comparison between the two systems fair and justify our point of view, the following lemma is being formulated:

Lemma 1: In a perfect spectrum-slicer FBG with total spectral width of $\Delta \lambda$ within a certain transmission optical fiber window, assume that the spectral width of any slice is $\delta \lambda$, where $\delta \lambda \ll \Delta \lambda$. If this FBG is used in a SAC-OCDMA system with MAI cancellation, then the number of available users adopting Hadamard codewords is given by

$$
N=\frac{\Delta \lambda}{\delta \lambda}-1 \cong \frac{\Delta \lambda}{\delta f \cdot \lambda_{0}^{2} / c}-1
$$

where $\lambda_{0}$ is the central wavelength, $c$ is the speed of light, and $\delta f$ is the corresponding bandwidth of a slice in frequency domain. On the other hand, if this FBG is used in WDM system adopting NRZ coding formats, then the number of available users is given by

$$
N_{W} \cong \frac{\Delta \lambda}{(R+\delta f) \cdot \lambda_{0}^{2} / c}
$$

where $R$ is the transmission rate of a user in bits/s.

Proof: The first assertion is immediate from Fig. 4(b) and the properties of Hadamard codes. The second assertion can be proved as follows:

Case 1: If $\delta f=0$ : This case is ideal one (not practical), where a spectral slice approaches a single wavelength. By noticing that each user in a WDM system modulates a single wavelength, each of these wavelengths would have sidebands of at least $R \mathrm{~Hz}$ in frequency domain after modulation. Converting these side bands into wavelength domain, each wavelength would have sidebands of at least $R \cdot \lambda_{0}^{2} / c$.

Case 2: If $\delta f>0$ : This case is a practical one, where a spectral slice contains a band of wavelengths. By noticing that each user in a WDM system modulates a single spectral slice, it is easy to check that in this case each modulated spectral slice would cover a band of at least $R+\delta f$ in frequency domain. Converting these bands into wavelength domain, each modulated spectral slice would cover a band of at least $(R+\delta f) \cdot \lambda_{0}^{2} / c$.

Remark 1: In case of SAC-OCDMA, all spectral slices can be used; however in case of WDM, only portion of the available wavelengths (slices) can be used because of the necessity to use guard band between different wavelengths (slices) to allow for the modulation sidebands, Fig. 4(a).

Remark 2: In case of WDM, it is obvious that as the transmission rate $R$ increases, the number of useable wavelengths (slices) decreases because of the increase of the size of the sidebands. However, in case of SAC-OCDMA, the number of available codewords remains the same. 


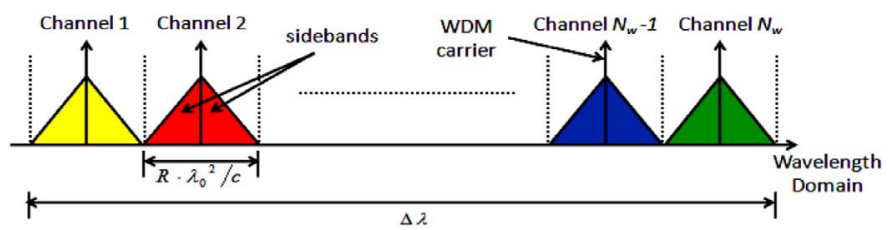

(a)

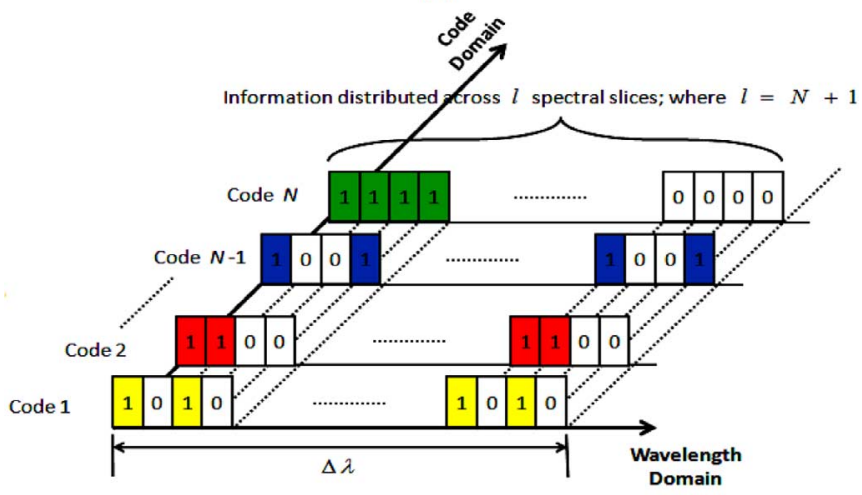

(b)

Fig. 4. Number of available channels in (a) WDM (b) SAC.

Remark 3: There are two interesting extreme cases:

1. If $R \gg \delta f$, then $N \gg N_{W}$;

2. If $R \ll \delta f$, then $N \approx N_{W}$.

Remark 4: We give here a numerical example to illustrate our point of view. Assume a band of operation $\Delta \lambda=20 \mathrm{~nm}$, a slice width of $\delta \lambda=20 / 128=0.15625 \mathrm{~nm}$ and a central wavelength of $\lambda_{0}=1550 \mathrm{~nm}$ for both SAC and WDM systems. In addition, assume a bit rate of $R=40 \mathrm{Gbps}$ for each single user. This means that the minimum required bandwidth for transmitting each individual user's information is $40 \mathrm{GHz}$ (or equivalently $0.32 \mathrm{~nm}$ linewidth) assuming on-off keying nonreturn-to-zero (OOK-NRZ) line coding is employed. In case of WDM, the maximum number of channels $N_{W}$ that can be allocated across the $20 \mathrm{~nm}$ band is $20 /(0.32+0.15625) \cong 41$ channels assuming no guard bands are left at all, i.e., allowing for sharp filters (perfect FBGs) to be used. Furthermore, if we consider the very limiting case with a slice width of $\delta \lambda=0$ (single wavelength per user), we get $N_{W}=20 /(0.32+0) \cong 62$. This means that increasing $N_{W}$ beyond 62 is not allowed as it will cause the information carried out on each channel or carrier to spill over the neighboring channels, i.e., crosstalk will result. Conversely, in case of SAC, this is not the case as the 40 Gbps information is equally distributed among the spectral slices or subchannels across the whole $20 \mathrm{~nm}$ band. This means that we can set the number of available codes or channels $N=20 / 0.15625-1=$ 127 , which is not far from the values assumed by researchers in [13] and [19], as long as we are assuming perfect FBGs are used for encoding/decoding process. Furthermore, if we consider the very limiting case with a slice width of $\delta \lambda=0$, we can increase $N$ as we wish.

Concluding what was previously discussed, as long as we are assuming perfect hardware implementation in both systems, SAC is better than WDM in terms of utilizing the available spectrum taking into account that we are employing perfect MAI cancellation in our system. In other words, the maximum the-

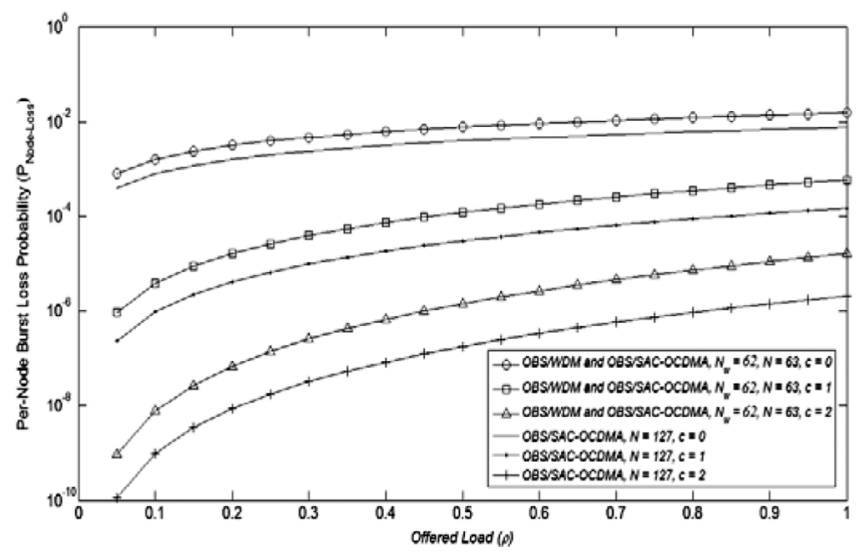

Fig. 5. Semi-log plot for MAC layer per-node burst loss probability versus the Offered Load for OBS/SAC-OCDMA and OBS/WDM systems at different number of code and wavelength converters.

oretical limit provided by SAC is higher than that of WDM. Added to that, SAC-OCDMA is also advantageous over WDM in terms of providing more privacy. Of course in real systems, the aforementioned advantages of our proposed system are acquired at the expense of increasing complexity as compared to WDM systems.

\section{RESULTS}

Based on Lemma 1 and employing (3) and (4), the per-node burst loss probability $P_{\text {Node-Loss }}$ is plotted in Fig. 5 versus the offered load $\rho$ in order to compare between OBS/SAC-OCDMA and OBS/WDM systems at different number of code and wavelength converters. We assume an average burst length $L_{b}$ of 10 Mbits. When using no, one and two converters in both systems, results reveal that employing SAC-OCDMA in the optical layer has a significant advantage over the ordinary WDM in terms of lower $P_{\text {Node-Loss }}$ for all traffic conditions. Moreover, we observe that the difference between the two systems is much more evident in the case of presence of conversion capabilities. This is due to the better utilization for the extra number of servers, i.e., the difference between the number of codes and wavelengths, by employing converters noting that code conversion imposes less complex implementation requirements when compared to wavelength conversion.

Another observation can be made from Fig. 5 is that having code conversion capability can improve the system performance in terms of lower Per-Node Loss Probability. We should notice that for low traffic values, the code conversion effectiveness is superior when comparing it with the case of high traffic values. This is because when the traffic arrival rate is low; there will be more free codes to which the contending bursts can be converted.

Utilizing the results obtained in (5), (9) and (10), Fig. 6 shows $P_{\mathrm{MAC}-\text { Loss }}$, BurstER $\mathrm{PHY}_{\text {and }}$ BurstER $\mathrm{Bverall}_{\text {versus }}$ the number of codes $N$ deployed in an OBS/SAC-OCDMA system having one code converter. Furthermore, values of BurstER $_{\mathrm{PHY}}$ are calculated when the threshold value $\mathrm{Th}$ is set to its optimum value which is found via a genetic algorithm based optimization that minimizes BurstER ${ }_{\mathrm{PHY}}$. In Fig. 6, it is apparent that $P_{\mathrm{MAC}-\text { Loss }}$ decreases when using larger 


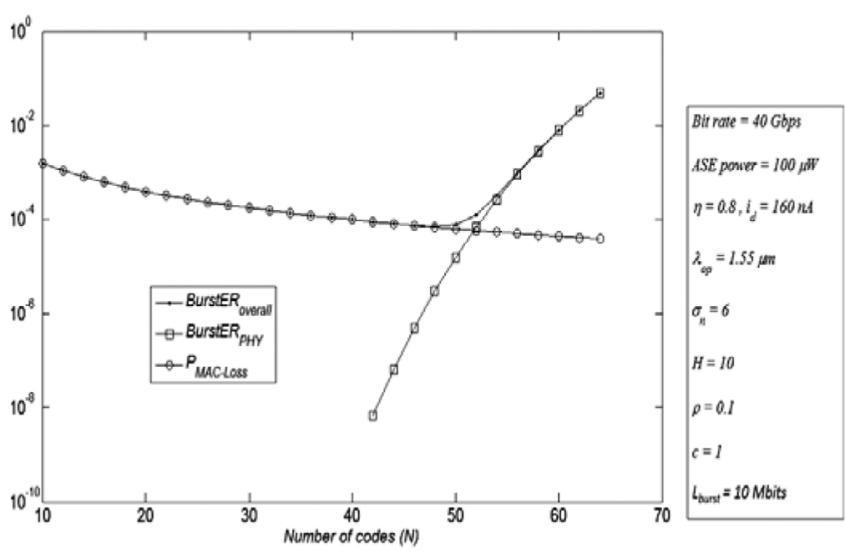

Fig. 6. Semi-log plot of MAC burst loss probability, optical layer burst error rate and overall burst error rate versus the available number of codes for OBS/ SAC-OCDMA system with one code converter.

number of codes because it is more probable for a $\mathrm{CP}$ to succeed in the reservation process when the available number

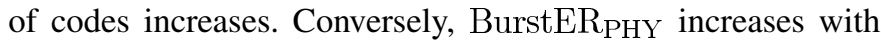
the increase of the number of codes $N$ which is justified by the presence of the 1: $N$ splitter at the front end of the egress node; hence, using more codes enlarges the splitting ratio leading to a lower received power level incident on the photodetectors which raises the probability for decision errors at the egress node. Combining both $P_{\mathrm{MAC}-\text { Loss }}$ and BurstER $\mathrm{PHY}_{\mathrm{PH}}$, we find

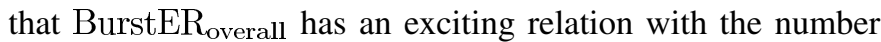
of codes $N$. For small values of $N, P_{\text {MAC-Loss }}$ dominates the overall error performance as compared to BurstER $\mathrm{PHY}_{\mathrm{PH}}$;

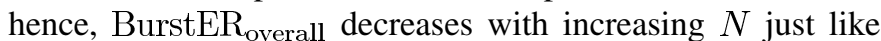
the behavior of $P_{\mathrm{MAC}-\text { Loss }}$. On the other hand, for large values

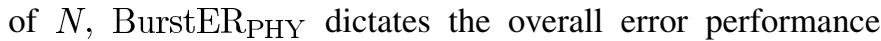
forcing BurstER $R_{\text {overall }}$ to increase with the increasing value of $N$. Intuitively, there is a minimum value for BurstER $\mathrm{B}_{\text {overall }}$ which is clearly the optimum design point, i.e., one should set the number of codes used in the system to the proper value that results in the least achievable BurstER overall $_{\text {. }}$

Next, Fig. 7 is a surface representing BurstER overall as a $_{\text {a }}$ function of both the number of codes $N$ and the offered load $\rho$ for the OBS/SAC-OCDMA system at different number of code converters. For each value of $c$, it is obvious that there always exists an optimum value for $N$ at each $\rho$ leading to the

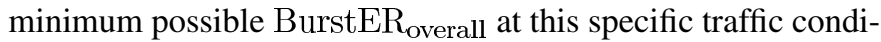
tion when $c$ code converters are used. Moreover, the efficacy of code conversion is quite evident from this figure as it is clear that introducing one more code converter enhances the overall performance of the system by decreasing BurstER $_{\text {overall }}$ significantly. In addition, we observe that this reduction in

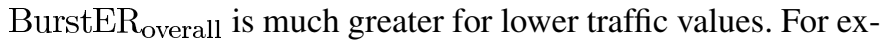
ample, at $N=10$ and $\rho=0.1$, BurstER overall $=10^{-1.024}$ for $c=0$ and BurstER overall $=10^{-2.807}$ for $c=1$. On the other hand, at $N=10$ and $\rho=1$, BurstER overall $=10^{-0.2115}$ for $c=0$ and BurstER $\mathrm{overall}=10^{-0.775}$ for $c=1$. Another issue concerning Fig. 7 is that the optimum number of codes $N_{\text {opt }}$ decreases clearly when more code converters are employed. For example, at $\rho=0.05, N_{\text {opt }}=46,42$, and 40 for $c=1,2$ and 3 respectively. This observation is justified by the fact that adding

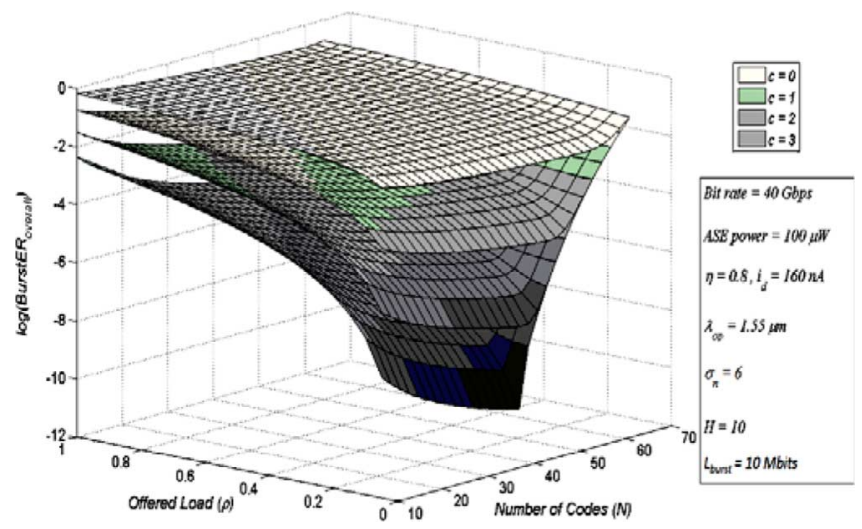

Fig. 7. Log overall burst error rate versus both the available number of codes and the offered load for OBS/SAC-OCDMA system at different number of code converters.
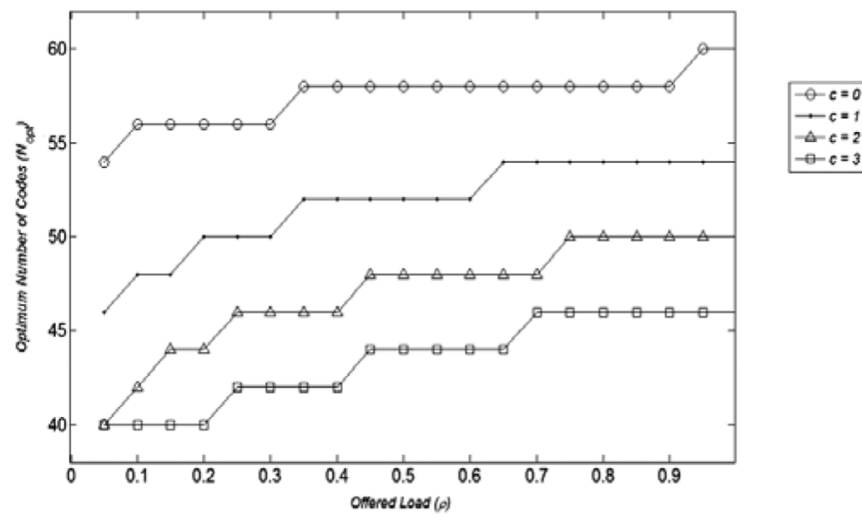

Fig. 8. Optimum number of codes versus the offered load at different number of code converters employed.

more code converters decreases $P_{\mathrm{MAC}-\text { Loss }}$ which shortens the range of dominance of $P_{\mathrm{MAC}-\text { Loss }}$ over BurstER $\mathrm{PHY}_{\mathrm{PH}}$ leading to a smaller value of $N_{\text {opt }}$.

At last, Fig. 8 shows the optimum number of codes $N_{\text {opt }}$ targeted for system design against the offered load $\rho$ for different values of code converters $c$. It is clear that $N_{\text {opt }}$ is larger for heavier traffic conditions. This is due to the dominance of the $P_{\text {MAC-Loss }}$ over BurstER $R_{\mathrm{PHY}}$ for heavier traffic values which corroborates the effectiveness of adding more codes in order to reduce $P_{\text {MAC-Loss }}$ (the dominant error component). Another interesting observation from Fig. 8, given a certain number of code converters used, the value of $N_{\text {opt }}$ remains constant for a certain range of $\rho$, which is very promising because the value of $N_{\mathrm{opt}}$ at which the system should be operated at a certain traffic condition remains also optimum when the traffic conditions change slightly, i.e., achieving a robust design for our system in terms of $N_{\text {opt }}$ is feasible.

\section{CONCLUSION}

We propose a new contention resolution technique for OBS networks by employing SAC-OCDMA instead of WDM in the optical layer underneath OBS in the MAC layer. Results show that the newly proposed OBS/SAC-OCDMA system outperforms the previous OBS/WDM system in terms of per-node burst loss probability. In addition, employing code converters 
enhances the proposed system performance noticeably especially for low traffic scenarios. Furthermore, an all-optical MAI cancellation is proposed. Finally, we observe that the overall burst error rate of the system is minimum at certain values for the employed number of codes which are the optimum points at which the system should be operated.

Considering the complexity of the proposed system, employing code converters is contingent on the availability of tunable chirped FBGs which are practically available as reported in [14] and [15]. Furthermore, the tuning speed of chirped FBGs is also an important design issue in our proposed system. Typical values for the tuning speed of chirped FBGs are in the order of few milliseconds which seems quite convenient with typical values of DB duration. For example, in our numerical results, the DB length is 10 Mbits and the bit rate is $40 \mathrm{Gbps}$, hence the DB duration will be $0.25 \mathrm{~ms}$. Finally, it should be noted that we can slightly reduce the bit rate to be more convenient with slower tuning speeds of chirped FBGs, i.e., there is a trade-off between the level of technology required and the transmission bit rate.

Another issue related to system complexity is the practical limitation imposed by the variable threshold optical limiters on our proposed system; however, researchers in [16] reported the availability of such devices. More efforts are expected to realize practical variable threshold limiters in the near future.

Finally, our proposed system is also disadvantageous somehow in that it requires an ASE broadband source at each node. Such active elements required increase the overall power requirement in the network; however, ASE broadband sources with large output power levels are practically available. Furthermore, the power required from each ASE source in the core node increases linearly with the deployed number of converters in the node, which is the price paid for the tremendous improvement achieved in the overall system performance by adding code converters. Despite the proportionality between the total power requirement and the number of code converters, we should note that a tiny number of code converters can achieve a huge improvement in the performance as the numerical results suggested, while at the same time having a small impact on the overall power requirement in the network.

\section{FUTURE WORK}

We should note that this work is a preliminary work in this area. We have studied using SAC-OCDMA as the only multiple access technique in the PHY layer in OBS networks. Future research avenues in this area can be directed towards combining both WDM and SAC-OCDMA in the PHY layer in OBS networks in order to provide much more available number of channels, hence resolving more contentions. Some research work can be found in other aspects proposing to employ hybrid WDM and spectral-encoding OCDMA for FTTH netwroks such as in [17]. Also in [19], the author proposes using SAC-OCDMA combined with WDM to improve SAC-OCDMA. Finally in [18], researchers propose to use time-spread OCDMA over WDM for PONs as a solution to Gigabit symmetric FTTH networks.

Also, it should be noted that this paper proposed using spectral-encoding OCDMA based on encoding the amplitude of the spectral components in OBSNs; however, further research can be carried out in this area to test different types of spectral-encoding OCDMA that are based on phase encoding and compare it to the currently proposed technique.

\section{REFERENCES}

[1] C. Qiao and M. Yoo, "Optical burst switching (OBS)—A new paradigm for an optical internet,” J. High Speed Netw., vol. 8, no. 1, pp. 69-84, Jan. 1999

[2] I. Baldine, G. N. Rouskas, H. G. Perros, and D. Stevenson, "JumpStart: A just-in-time signaling architecture for WDM burst-switched networks," IEEE Commun. Mag., vol. 40, no. 2, pp. 82-89, Feb. 2002.

[3] T. Battestilli and H. Perros, "An introduction to optical burst switching," IEEE Commun. Mag., vol. 41, no. 8, pp. S10-S15, Aug. 2003.

[4] X. Yu, J. Li, X. Cao, Y. Chen, and C. Qiao, "Traffic statistics and performance evaluation in optical burst switched networks," J. Lightw. Technol., vol. 22, no. 12, pp. 2722-2738, Dec. 2004.

[5] X. Yu, Y. Chen, and C. Qiao, "Study of traffic statistics of assembled burst traffic in optical burst switched networks," in Proc. Opticomm., 2002, pp. 149-159

[6] S. Oh and M. Kang, "A burst assembly algorithm in optical burst switching networks," in Proc. Opt. Fiber Commun. Conf., 2002, pp. $771-773$.

[7] K. Kamakura, O. Kabranov, D. Makrakis, and I. Sasase, "OBS networks using optical code division multiple access techniques," in Proc. IEEE Int. Conf. on Commun. (ICC'04), Paris, France, Jun. 2004, pp. 1725-1729.

[8] M. Kavehard and D. Zaccarin, "Optical code-division-multiplexed systems based on spectral encoding of noncoherent sources," J. Lightw. Technol., vol. 13, no. 3, pp. 534-545, Mar. 1995.

[9] A. Grunnet-Jepsen, A. E. Johnson, E. S. Maniloff, T. W. Mossberg, M. J. Munroe, and J. N. Sweetser, "Fiber Bragg grating based spectral encoder/decoder for lightwave CDMA," Electron. Lett., vol. 35, pp. 1096-1097, 1999.

[10] X. Zhang et al., "All-optical AND gate at $10 \mathrm{Gbit} / \mathrm{s}$ based on cascaded single-port-couple SOAs," Opt. Expr., vol. 12, no. 3, pp. 361-366, 2004.

[11] N. Akar, E. Karasan, and K. Dogan, "Wavelength converter sharing in asynchronous optical packet/burst switching: An exact blocking analysis for Markovian arrivals," IEEE J. Sel. Areas Commun., vol. 24, no. 12, pp. 69-80, Dec. 2006.

[12] R. M. Gagliardi and S. Karp, Optical Commun.. New York, NY: John Wiley, 1995.

[13] E. Smith, R. Blaikie, and D. Taylor, "Performance enhancement of spectral-amplitude-coding optical CDMA using pulse-position modulation," IEEE Trans. Commun., vol. 46, no. 9, pp. 1176-1185, 1998.

[14] S. Y. Set, B. Dabarsyah, C. S. Goh, K. Katoh, Y. Takushima, and K. Kikuchi, "A widely tunable fiber Bragg grating with a wavelength over 40 nm," in Proc. Opt. Fiber Commun. Conf. (OFC), 2001, vol. 1, pp. MC4-1-MC4-3.

[15] Y. Painchaud, C. Paquet, and M. Guy, "Optical tunable dispersion compensators based on thermally tuned fiber Bragg gratings," OSA Opti. Photon. News, vol. 18, no. 9, Sep. 2007.

[16] H. Goto, T. Konishi, and K. Itoh, "Unitary variable threshold optical limiter without bulky optical systems," presented at the IEEE Conf. on Lasers and Electro-Optics, Pacific Rim, 2007.

[17] J. Huang, Y. Chang, and D. Wang, "Hybrid WDM and optical CDMA implemented over waveguide-grating-based fiber-to-the-home networks," Opt. Fiber Technol., vol. 13, no. 3, pp. 215-225, Jul. 2007.

[18] K. Kitayama, X. Wang, and N. Wada, "OCDMA over WDM PONsolution path to gigabit-symmetric FTTH," $J L T$, vol. 24, no. 4, pp. 1654-1662, Apr. 2006.

[19] C. Yang, "Hybrid wavelength-division-multiplexing/spectral-amplitude-coding optical CDMA system," IEEE Photon. Technol. Lett., vol. 17 , no. 6, Jun. 2005. 


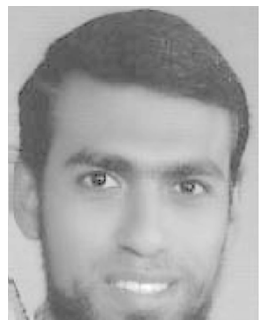

Mohammad Y. S. Sowailem (S'05) was born in Yanbu, Saudi Arabia, in 1984. He received the B.S. degree in electrical engineering from Alexandria University, Alexandria, Egypt, in 2006. He is currently working towards the M.S. degree at the Department of Electrical Engineering, Alexandria University, Alexandria, Egypt.

In 2006, he joined the Basic and Applied Science Department, Arab Academy of Science and Technology, as a part time Teaching Assistant for one year. Then, he joined SySDSoft, Inc. as a DSP Engineer in August 2007 till now. His current research interests include optical burst switched networks (OBSNs), optical code-division multiple-access (OCDMA) networks, media access control (MAC) protocols in optical networks, computer networks, and wireless communications.

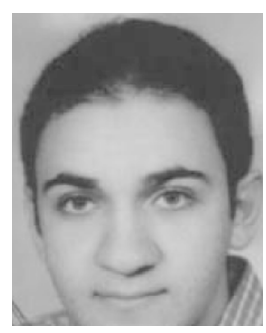

Mohamed H. S. Morsy (S'07) was born in Cairo, Egypt, in 1985. He received the B.S. degree in electrical engineering from Alexandria University, Alexandria, Egypt, in 2006. He is currently working towards the M.S. degree at the Department of Electrical Engineering, Alexandria University, Alexandria, Egypt.

In 2006, he joined the Department of Electrical Engineering, Alexandria University, as a Teaching Assistant for three years until now. His current research interests include optical burst switched networks (OBSNs), optical code-division multiple-access (OCDMA) networks, media access control (MAC) protocols in optical networks, computer networks, and wireless communications.

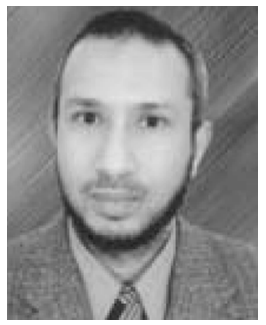

Hossam M. H. Shalaby (S'83-M'91-SM'99) was born in Giza, Egypt, in 1961. He received the B.S. and M.S. degrees from the University of Alexandria, Egypt, in 1983 and 1986, respectively, and the Ph.D. degree from the University of Maryland, College Park, MD, in 1991, all in electrical engineering.

In 1991, he joined the Department of Electrical Engineering, University of Alexandria, Egypt, as an Assistant Professor. He was promoted to the position of Associate Professor in 1996 and then to Professor in 2001 (current position). From March to April 1996, he was a Visiting Professor at the Electrical Engineering Department, Beirut Arab University, Lebanon. From December 2000 to 2004, he was an Adjunct Professor with Department of Electrical and Information Engineering, Faculty of Sciences and Engineering, Laval University, Quebec, Canada. From February to May 2009, he was an Adjunct Professor at the Electronics Engineering Department, American University in Cairo, Egypt. From September 1996 to February 2001, he was on leave from the University of Alexandria, where he was with two of the following places. From September 1996 to January 1998, he was an Associate Professor with the Electrical and Computer Engineering Department, International Islamic University Malaysia, and from February to December 1998, he was with the School of Electrical and Electronic Engineering, Nanyang Technological University, Singapore, where he was a Senior Lecturer and from January 1999 to February 2001, an Associate Professor. His research interests include optical communications, optical CDMA, optical burst switching, OFDM technology and information theory.

Dr. Shalaby received SRC fellowship from 1987 to 1991 (Systems Research Center, MD), State Excellence Award in Engineering Sciences, in 2007 (Academy of Scientific Research and Technology, Egypt), Shoman Prize for Young Arab Researchers in 2002 (The Abdul Hameed Shoman Foundation, Amman, Jordan), State Incentive Award in Engineering Sciences, twice in 1995 and 2001 (Academy of Scientific Research and Technology, Egypt), University Incentive Award in 1996 (University of Alexandria, Egypt), and Soliman Abd-El-Hay Award in 1995 (Academy of Scientific Research and Technology, Egypt). He has served as a Student Branch Counselor (Alexandria University), IEEE Alexandria and North Delta Subsection, from 2002 to 2006, and served as a Chairman of the Student Activities Committee of IEEE Alexandria Subsection from 1995 to 1996. 